\title{
Topology Optimization for a Dielectric Optical Cloak Based on an Exact Level Set Approach
}

\section{$\operatorname{AUTHOR}(\mathrm{S}):$}

Yamada, Takayuki; Watanabe, Hayato; Fujii, Garuda; Matsumoto, Toshiro

\section{CITATION:}

Yamada, Takayuki ...[et al]. Topology Optimization for a Dielectric Optical Cloak Based on an Exact Level Set Approach. IEEE Transactions on Magnetics 2013, 49(5): 2073-2076

\section{ISSUE DATE:}

2013-05

URL:

http://hdl.handle.net/2433/174317

\section{RIGHT:}

(c) 2013 IEEE. Personal use of this material is permitted. Permission from IEEE must be obtained for all other uses, in any current or future media, including reprinting/republishing this material for advertising or promotional purposes, creating new collective works, for resale or redistribution to servers or lists, or reuse of any copyrighted component of this work in other works.; この論文は出版社版でありません。引用の際には出版社版をご確認ご利用ください。; This is not the published version. Please cite only the published version. 


\title{
Topology Optimization for a Dielectric Optical Cloak Based on an Exact Level Set Approach
}

\author{
Takayuki Yamada $^{1}$, Hayato Watanabe ${ }^{2}$, Garuda Fujii ${ }^{3}$ and Toshiro Matsumoto ${ }^{2}$ \\ ${ }^{1}$ Department of Mechanical Engineering and Science, Kyoto University Kyoto, 606-8501, JAPAN \\ ${ }^{2}$ Department of Mechanical Science and Engineering, Nagoya University, Nagoya, 464-8603, JAPAN \\ ${ }^{3}$ Department of Machine Intelligence and Systems Engineering, Akita Prefectural University, Akita, 015-0055, JAPAN
}

\begin{abstract}
This paper proposes a topology optimization method for a dielectric optical cloak that provides results that are perfectly free from intermediate materials, based on a level set boundary expression and the Finite Element Method. The finite element mesh is regenerated to fit the iso-surface of the level set function at every iterative step, to remove intermediate materials, so that the obtained optimal structure consists of only two materials, the dielectric material and air. First, the level set-based topology optimization is formulated and a topology optimization algorithm is proposed for the exact level set approach. Next, design requirements for the dielectric optimal cloak device are clarified and an objective functional for the design is formulated. The proposed method is then applied to a simple numerical problem to illustrate its effectiveness.
\end{abstract}

Index Terms — Finite element method, level set method, optical cloak, optimum design, topology optimization

\section{INTRODUCTION}

$\mathrm{O}$ PTICAL cloaks are able to make an object unobservable by wrapping it with a specific frequency range of electromagnetic waves. The configuration of an optical cloak can be given theoretically by using transformation optics [1] but the theoretical material properties are subject to continuous change and extremely high value. The required material properties make practical realization problematic.

On the other hand, an optimum design method for dielectric optical cloaks has recently been proposed [2], using a densitybased topology optimization method in which the optimal structure is represented using a continuous density distribution that allows intermediate material, so-called grayscale areas. Although grayscale areas can be interpreted as being microporous in this case, such optimal structures cannot be manufactured.

To overcome the problem of grayscales, level set basedtopology optimization methods have been proposed [3] and extended to several design problems, such as thermal problems [4], motor design problem [5], magnet actuator design problem [6], and the design of electro-metamaterials [7]. In these methods, the structural boundaries are represented by the iso-surface of the level set function [8], and the level set function is updated based on a reaction-diffusion equation. The optimal configurations obtained by these methods are therefore smooth and clear. In addition, level set-based methods can easily incorporate consideration of various geometrical constraints so that appropriate results from a manufacturing standpoint can be obtained.

This paper proposes a new topology optimization method for a dielectric optical cloak, based on the level set method and marching cube concept [9], which perfectly eliminates grayscales. The new aspect presented here is that the finite element mesh is regenerated to fit the iso-surface of the level set function, which represents the structural boundaries, at

Manuscript received October 31, 2012 (date on which paper was submitted for review). Corresponding author: Takayuki Yamada (e-mail: takayuki@me.kyoto-u.ac.jp).

Digital Object Identifier inserted by IEEE every iterative step, using the marching cube concept. Consequently, the obtained configuration is perfectly free from intermediate materials. In this paper, a level set-based topology optimization method that incorporates a fictitious interface energy model [3] is used to obtain smooth optimal structural boundaries.

\section{FORMULATIONS}

\section{A Level set-based topology optimization}

The level set function is introduced to represent the boundaries between dielectric material and air that express the structural configuration. In this level set-based topology optimization, the level set function is defined as follows:

$$
\left\{\begin{array}{lll}
0<\phi(\boldsymbol{x}) \leq 1 & \text { if } & \forall \boldsymbol{x} \in \Omega \backslash \partial \Omega \\
\phi(\boldsymbol{x})=0 & \text { if } & \forall \boldsymbol{x} \in \partial \Omega \\
-1 \leq \phi(\boldsymbol{x})<0 & \text { if } & \forall \boldsymbol{x} \in D \backslash \Omega
\end{array}\right.
$$

where $\Omega$ is a material domain and $D$ is the fixed design domain. Here, upper and lower limits of the level set function are imposed since the regularization term derived from the phase field model concept [10] is defined using the magnitude of the gradient of the level set function. In addition, the characteristic function is defined by

$$
\chi(\phi)=\left\{\begin{array}{lll}
1 & \text { if } \quad \phi(x) \geq 0 \\
0 & \text { if } \quad \phi(x)<0 .
\end{array}\right.
$$

The level set-based topology optimization problem is formulated as follows:

$$
\inf _{\phi} F[\chi(\phi)]=\int_{\Omega_{o b j}} f(\boldsymbol{x}) \mathrm{d} \Omega,
$$

where $F$ is an objective functional, $f(\boldsymbol{x})$ is a distributed function representing the objective functional, and $\Omega_{\mathrm{obj}}$ is a domain defined in the objective functional. Next, a fictitious interface energy is introduced to the objective functional to regularize the topology optimization problem:

$$
\inf _{\phi} \quad F_{R}[\chi(\phi)]=F[\chi(\phi)]+R[\phi],
$$


where $F_{R}$ is the regularized objective functional and $R$ is a regularization term representing the use of a regularization parameter $\tau$ as follows:

$$
R[\phi]=\int_{D} \frac{1}{2} \tau|\nabla \phi(\boldsymbol{x})|^{2} \mathrm{~d} \Omega .
$$

Next, the Karush-Kuhn-Tucker (KKT) condition of the topology optimization problem is derived as follows:

$$
F_{R}^{\prime}=0
$$

A level set function satisfying this condition is a candidate optimal solution, but such solutions are extremely difficult to obtain directly. In the proposed method, a fictitious time $t$ is introduced and a time-evolutionary equation for the level set function is derived. We assume that gradient of the level set function is proportional to its time derivative, as follows:

$$
\frac{\partial \phi(\boldsymbol{x}, t)}{\partial t}=-K F_{R}^{\prime}(\phi, t),
$$

where $K$ is a positive coefficient of proportionality. Finally, the time evolutionary equation is derived as follows:

$$
\frac{\partial \phi(\boldsymbol{x}, t)}{\partial t}=-K\left(F^{\prime}(\phi, t)-\tau \nabla^{2} \phi(\boldsymbol{x}, t)\right) .
$$

We note that above equation is a type of reaction-diffusion equation and the derivative of the objective functional $F^{\prime}$ is defined as at the topological derivative [4].

\section{B Optimization algorithm}

The flowchart of the topology optimization is shown in Fig. 1. As shown, the level set function is first initialized. In the second step, the finite element mesh is generated based on the level set function and in the third step, the governing and adjoint equations are solved using the generated finite element mesh. Next, the sensitivity $F^{\prime}$ is computed and the level set function is updated based on Eq. 8. Here, if the objective functional is converged, the topology optimization process is finished; otherwise the procedure returns to the mesh generation process of the second step.

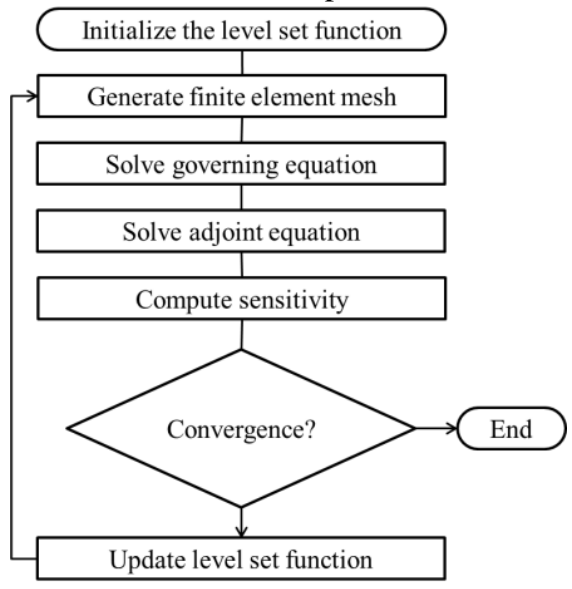

Fig. 1. Flowchart of the optimization procedure

We note that a novel aspect of the topology optimization algorithm is that the finite element mesh is regenerated every iteration, based on the marching cube concept [8], which perfectly eliminates intermediate materials. In the traditional marching cube method, the domain is divided into voxels, and the surface pattern of voxels is sorted into 14 different patterns, with each voxel identified based on the sign of the level set function at each voxel's eight vertices. In this paper, the marching cube concept is extended to a two-dimensional rectangular domain case using triangular elements, as shown in Fig. 2 where (a) and (b) represent material and void elements, respectively. Note that there are four patterns when material differences are considered. A finite element mesh that fits the iso-surface of the level set function is generated, based on the four cases illustrated in Fig. 2.

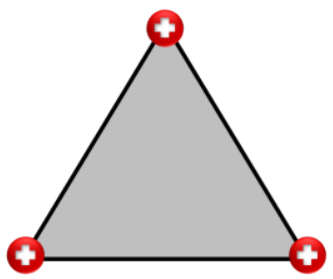

(a)

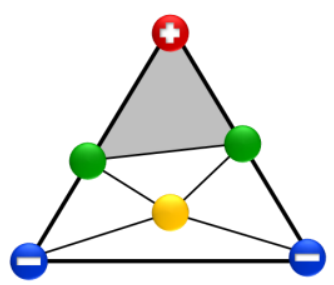

(c)

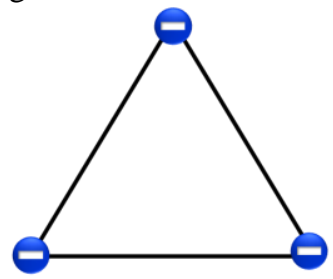

(b)

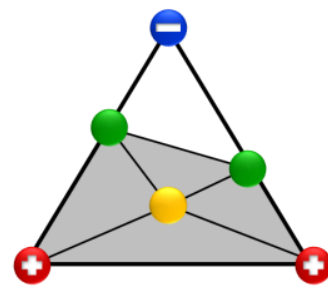

(d)

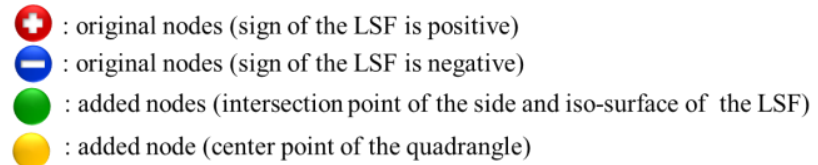

Fig. 2. Generated mesh patterns: (a) case1, (b) case 2, (c) case 3 , (d) case 4

\section{Optimization problem}

We now consider the design of an optical cloak device, based on the model shown in Fig. 3.

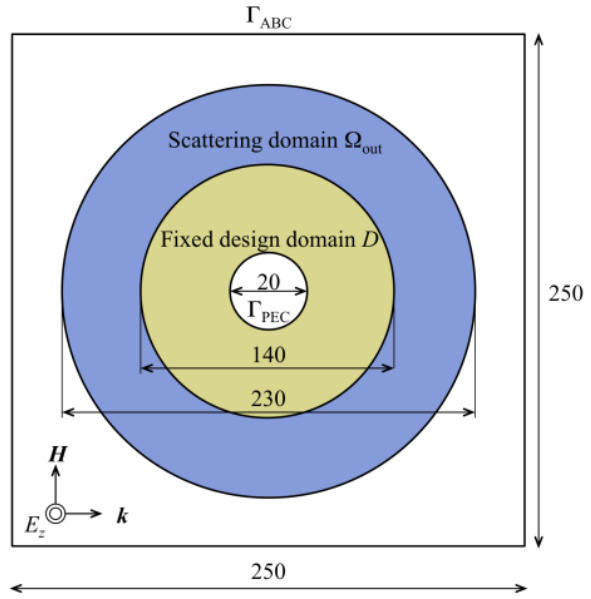

Fig.3. Design model of an optical cloak device

The circle at the center of the domain, $\Gamma_{\mathrm{PEC}}$, represents an ideal metallic material that obeys a perfect electric conductor condition $\Gamma_{\mathrm{PEC}}$. Here, incident waves $E_{i}$ enter the scattering 
domain from the left side:

$$
E_{i}=E_{0} e^{-j k_{0} \boldsymbol{k} \cdot \boldsymbol{r}},
$$

where $E_{0}$ is the amplitude of the incident wave, $j=\sqrt{-1}, k_{0}$ is the free space wave number, $\boldsymbol{k}$ is the normalized directional wave vector, and $\boldsymbol{r}$ is the position vector. The gray domain is the fixed design domain $D$ in which a material configuration consisting of a dielectric material and air is distributed. The domain exterior to the fixed design domain is the scattering domain, $\Omega_{\text {out }}$.

The optical cloak device must cause the effect of the ideal metallic material to disappear. That is, the optical cloak device should be designed to minimize the scattering field in the scattering domain. Therefore, the topology optimization of the design problem for the optical cloak is formulated as follows:

$$
\begin{array}{ll}
\inf _{\phi} \int_{\Omega_{\text {out }}} E_{s} E_{s}^{*} \mathrm{~d} \Omega \\
\text { subject to } \\
\nabla \cdot\left(\frac{1}{\mu_{r}} \nabla E_{s}\right)+k_{0}^{2} \epsilon_{r} E_{s}=-k_{0}^{2}\left(\epsilon_{r}-\frac{1}{\mu_{r}}\right) E_{i} \\
\begin{array}{ll}
\boldsymbol{n} \cdot \nabla E_{s}+j k_{0} E_{s}=0 & \text { in } D \cup \Omega_{o b j} \\
E_{s}=-E_{i} & \text { on } \Gamma_{A B C} \\
& \text { on } \Gamma_{P E C},
\end{array}
\end{array}
$$

where $E s$ is the scattering field, $E s^{*}$ is the complex conjugate of the scattering field, $\mu_{r}$ is the relative permeability, and $\varepsilon_{r}$ is an extended relative permittivity defined using the characteristic function as follows:

$$
\epsilon_{r}(\boldsymbol{x})=\left\{\begin{array}{lll}
\epsilon_{r}^{d i e} & \text { in } D & \text { if } \phi(\boldsymbol{x}) \geq 0 \\
\epsilon_{r}^{a i r} & \text { in } D & \text { if } \phi(\boldsymbol{x})<0 \\
\epsilon_{r}^{a i r} & \text { in } \Omega_{\text {out }}, &
\end{array}\right.
$$

where $\varepsilon_{r}^{d i r}$ and $\varepsilon_{r}^{a i r}$ are relative permittivity of the dielectric material and air, respectively.

\section{NUMERICAL EXAMPLE}

A numerical example is shown to confirm the utility of the proposed topology optimization method. The isotropic dielectric material has relative permeability $\varepsilon_{r}{ }^{d i r}=2.0$ and the wave number is set so that $k_{0}=9.5$. The domain size is set to $250 \times 250$. Note that the wave number and lengths are expressed using a dimensionless representation, in which the characteristic length is the mesh size of the fixed design domain. The number of degrees of freedom was 87,454, computational time was $2,128 \mathrm{sec}$ for each iteration (using 12 CPUs), and 546 iterations were performed. Figure 4 shows an initial design and the generated finite element mesh based on the initial level set function. The number of iteration is 546 . As shown in this figure, we confirm that the dielectric material domain and the air domain are perfectly separate.

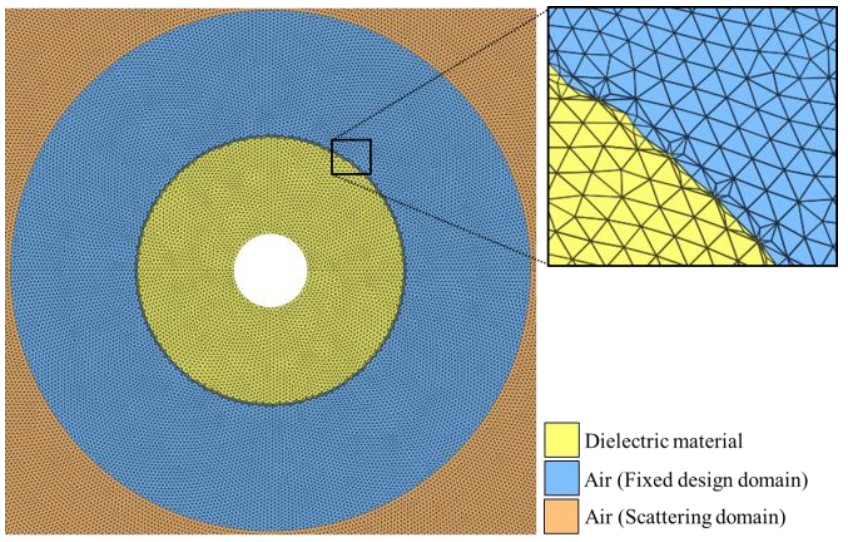

Fig. 4. Initial design and finite element mesh

Figure 5 shows an obtained configuration. As shown in the figure, the obtained optimal configuration is clear and smooth.

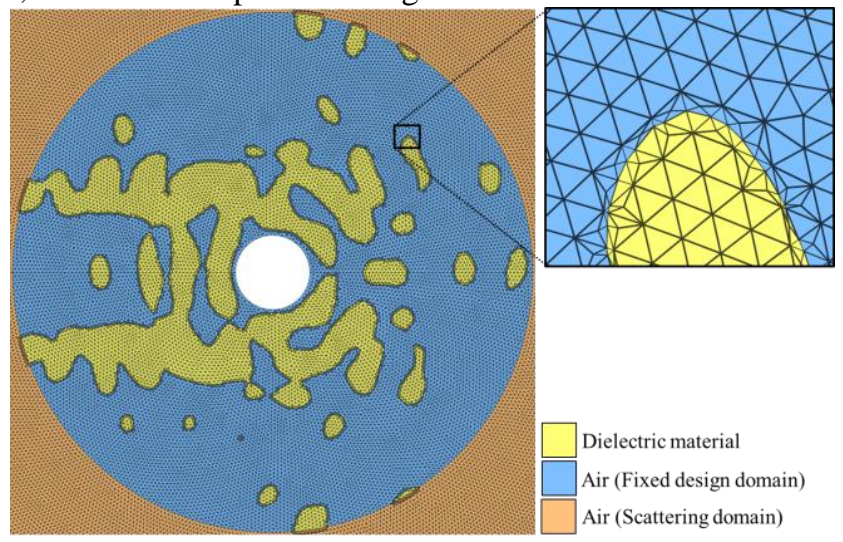

Fig. 5. Obtained design and finite element mesh

Figure 6 shows the total and scattering electric field of the case for the initial design and the obtained configuration. As shown, the scattering from the ideal metallic material at the center is reduced. The values of the objective functional for the optimal configuration and the configuration when free of dielectric material were 64.21 and 2,279, respectively. This result demonstrates that the optimization successfully found an appropriate dielectric configuration for the cloak device. 


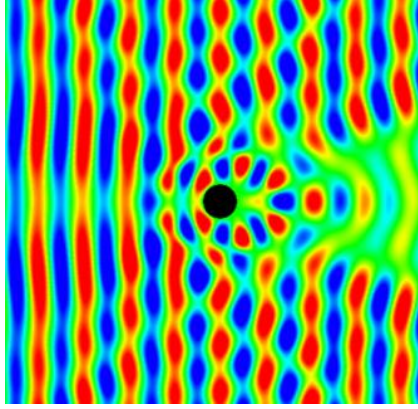

(a)

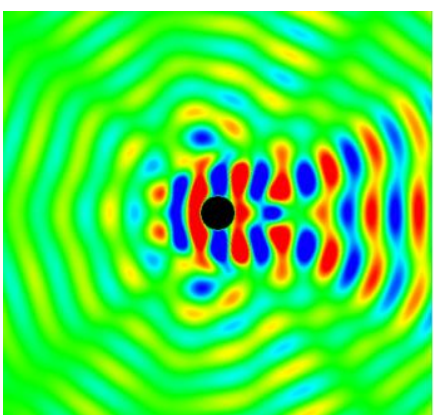

(c)

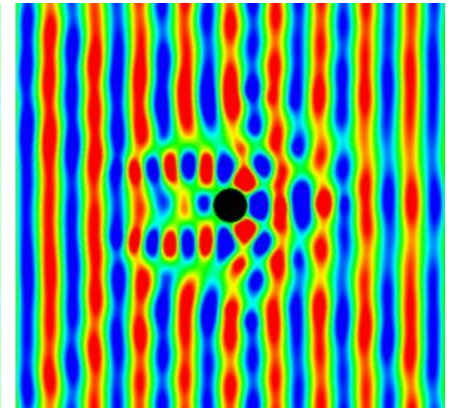

(b)

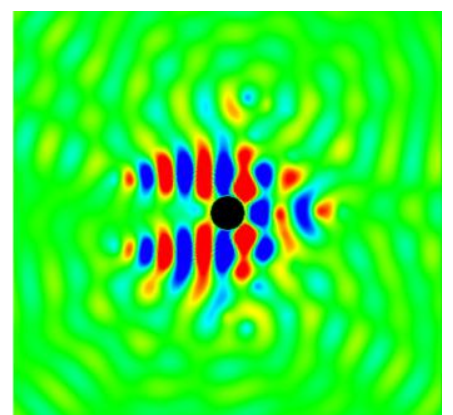

(d)
Fig. 6. Total and scattering electric field: (a) total electric field of the initial design, (b) total electric field of the obtained design, (c) scattering electric field of the initial design, (d) scattering electric field of the obtained design

\section{CONCLUSION}

This paper proposed a topology optimization method for a dielectric optical cloak based on an exact level set approach. We achieved the following:

1. The design problem requirements were clarified and an optimization problem was formulated based on the level set method.

2. To obtain a clear optimal configuration, an exact level set approach was constructed based on marching cube concepts.

3. To obtain an optimal configuration with smooth boundaries, a fictitious interface energy model was applied to the design problem.

4. We confirmed the utility of the proposed topology optimization method in a numerical example for a twodimensional optical cloak design problem.

5. It should be possible to extend the presented method to three-dimensional problems by using the original marching cube method.

\section{ACKNOWLEDGMENT}

This work was partially supported by a research grant from The Murata Science Foundation. The finite element mesh for the fixed design domain was constructed by using the "Altair Hyper Mesh" software package [11].

\section{REFERENCES}

[1] J. B. Pendry, D. Schurig and D. R. Smith, "Controlling electromagnetic fields," Science, vol.312, no.5781, pp.1780-1782, 2006.

[2] J. Andkjær and O. Sigmund, "Topology optimized low-contrast alldielectric optimal cloak," Appl. Phys. Lett., Vol.98, No.021112, 2011.

[3] T. Yamada, K. Izui, S. Nishiwaki and A. Takezawa, "A topology optimization method incorporating a fictitious interface energy," Comput. Meth. in Appl. Mech. and Eng., Vol. 199, No.45-48, pp. 2876$2891,2010$.

[4] T. Yamada, K. Izui and S. Nishiwaki, "A level set-based topology optimization method for maximizing thermal diffusivity in problems including design dependent Effects," J. of Mechanical Design, Vol.133, No.3, pp.031011.1-031011.9, 2011.

[5] S. Lim, S. Min and J. P. Hong, "Level-set-based optimal stator design of Interior permanent-magnet motor for torque ripple reduction using phase-field model," IEEE Trans. on Magn., Vol.47, No.10, pp.30203024, 2011.

[6] S. Lim and S. Min, "Design optimization of permanent magnet actuator using multi-phase level-set model," IEEE Trans. on Magn., Vol.48, No.4, pp.1641-1644.

[7] M. Otomori, T. Yamada, K. Izui, S. Nishiwaki and J. Andkjær, "A topology optimization method based on the level set method for the design of negative permeability dielectric metamaterials," Comput. Meth. in Appl. Mech. and Eng., Vol. 237-240, No.1, pp. 192-211, 2012.

[8] W. E. Lorensen and H. E. Cline, "Marching cubes: A high resolution 3D surface construction algorithm," ACM SIGGRAPH Comput. Graph., Vol.21, pp.136-169, 1987.

[9] S. Osher and J.A. Sethian, "Front propagation with curve dependent speed: algorithms based on the Hamilton-Jacobi formulations," $J$. Comput. Phys., Vol.78, pp.12-49, 1988.

[10] J.W. Cahn and J.E. Hilliard, Free energy of a nonuniform system I: Interfacial free energy," J. of Chem. Phys., Vol.28. No.2, pp.258-267, 1958.

[11] http://www.altairhyperworks.com/ 